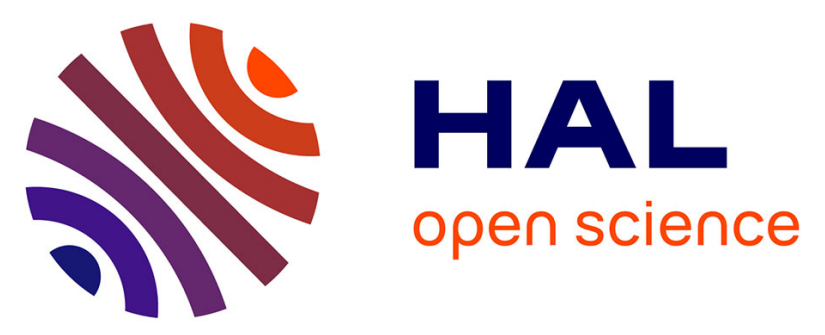

\title{
Combining Time-of-Flight Secondary Ion Mass Spectrometry Imaging Mass Spectrometry and CARS Microspectroscopy Reveals Lipid Patterns Reminiscent of Gene Expression Patterns in the Wing Imaginal Disc of Drosophila melanogaster
}

Florian Marty, Gianluca Rago, Donald F. Smith, Xiaoli Gao, Gert B. Eijkel, Luke Macaleese, Mischa Bonn, Erich Brunner, Konrad Basler, Ron M. A. Heeren

\section{To cite this version:}

Florian Marty, Gianluca Rago, Donald F. Smith, Xiaoli Gao, Gert B. Eijkel, et al.. Combining Time-of-Flight Secondary Ion Mass Spectrometry Imaging Mass Spectrometry and CARS Microspectroscopy Reveals Lipid Patterns Reminiscent of Gene Expression Patterns in the Wing Imaginal Disc of Drosophila melanogaster. Analytical Chemistry, 2017, 89 (18), pp.9664-9670. 10.1021/acs.analchem.7b00125 . hal-02317574

\author{
HAL Id: hal-02317574 \\ https://univ-lyon1.hal.science/hal-02317574
}

Submitted on 22 Jan 2021

HAL is a multi-disciplinary open access archive for the deposit and dissemination of scientific research documents, whether they are published or not. The documents may come from teaching and research institutions in France or abroad, or from public or private research centers.
L'archive ouverte pluridisciplinaire HAL, est destinée au dépôt et à la diffusion de documents scientifiques de niveau recherche, publiés ou non, émanant des établissements d'enseignement et de recherche français ou étrangers, des laboratoires publics ou privés.

\section{(1) (1) $\$$}

Distributed under a Creative Commons Attribution - NonCommercial - NoDerivatives 44.0 


\section{Combining Time-of-Flight Secondary lon Mass Spectrometry Imaging Mass Spectrometry and CARS Microspectroscopy Reveals Lipid Patterns Reminiscent of Gene Expression Patterns in the Wing Imaginal Disc of Drosophila melanogaster}

Florian Marty, ${ }^{\dagger, \#}$ Gianluca Rago, ${ }^{\ddagger}, \S$ Donald F. Smith, ${ }^{\ddagger}$ Xiaoli Gao, ${ }^{\|}$Gert B. Eijkel, ${ }^{\ddagger} \perp$ Luke MacAleese, ${ }^{\ddagger}, \nabla$

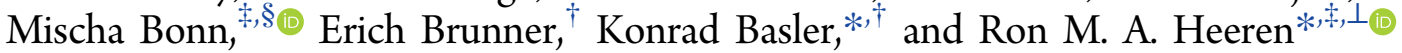

${ }^{\dagger}$ Institute of Molecular Life Sciences, University of Zürich, Winterthurerstrasse 190, CH-8057 Zürich, Switzerland

${ }^{\ddagger}$ FOM-Institute AMOLF, Science Park 104, 1098 XG Amsterdam, The Netherlands

${ }^{\S}$ Max Planck Institute for Polymer Research, Ackermannweg 10, 55128 Mainz, Germany

"Institutional Mass Spectrometry Laboratory, The University of Texas Health Science Center at San Antonio, 8403 Floyd Curl Drive, MC-7760 San Antonio, Texas, United States

${ }^{\perp}$ The Maastricht Multimodal Molecular Imaging Institute, Maastricht University, Universiteitssingel 50, 6229 ER Maastricht, The Netherlands

Supporting Information



ABSTRACT: Using label-free ToF-SIMS imaging mass spectrometry, we generated a map of small molecules differentially expressed in the Drosophila wing imaginal disc. The distributions of these moieties were in line with gene expression patterns observed during wing imaginal disc development. Combining ToF-SIMS imaging and coherent anti-Stokes Raman spectroscopy (CARS) microspectroscopy allowed us to locally identify acylglycerols as the main constituents of the pattern differentiating the future body wall tissue from the wing blade tissue. The findings presented herein clearly demonstrate that lipid localization patterns are strongly correlated with a developmental gene expression. From this correlation, we hypothesize that lipids play a so far unrecognized role in organ development.

$\mathrm{T}$ issue and organ development is governed by a multitude of signaling processes, patterning, and growth events. Model organisms such as Drosophila melanogaster are often used to study these developmental events and gain fundamental insight of organ development. The majority of pathways governing the development of Drosophila are evolutionarily conserved. ${ }^{1}$ Therefore, knowledge gained by studying this model organism's development can be transferred to other species such as humans.

The appendages of the adult fly such as wings, legs, or antenna develop from organ primordia called imaginal discs. ${ }^{2,3}$ The discs are subdivided into different compartments (e.g., dorsal, ventral, anterior, posterior compartments). The compartments are made of cells with distinct identities that do not mix. ${ }^{4-6}$ Compartmentalization is established in the early embryo, when the major body axes are determined. The compartment boundaries act as organizing centers that govern growth, patterning, and development of these discs. The boundaries define the expression of so-called morphogens, proteins which either act locally or diffuse over short and/or long ranges to pattern the growing organ. ${ }^{7-11}$ The identification of the molecules (classically proteins and RNAs) is often assigned to defined developmental processes due to their expression patterns or localizations to specific areas

Received: January 11, 2017

Accepted: July 20, 2017

Published: July 20, 2017 


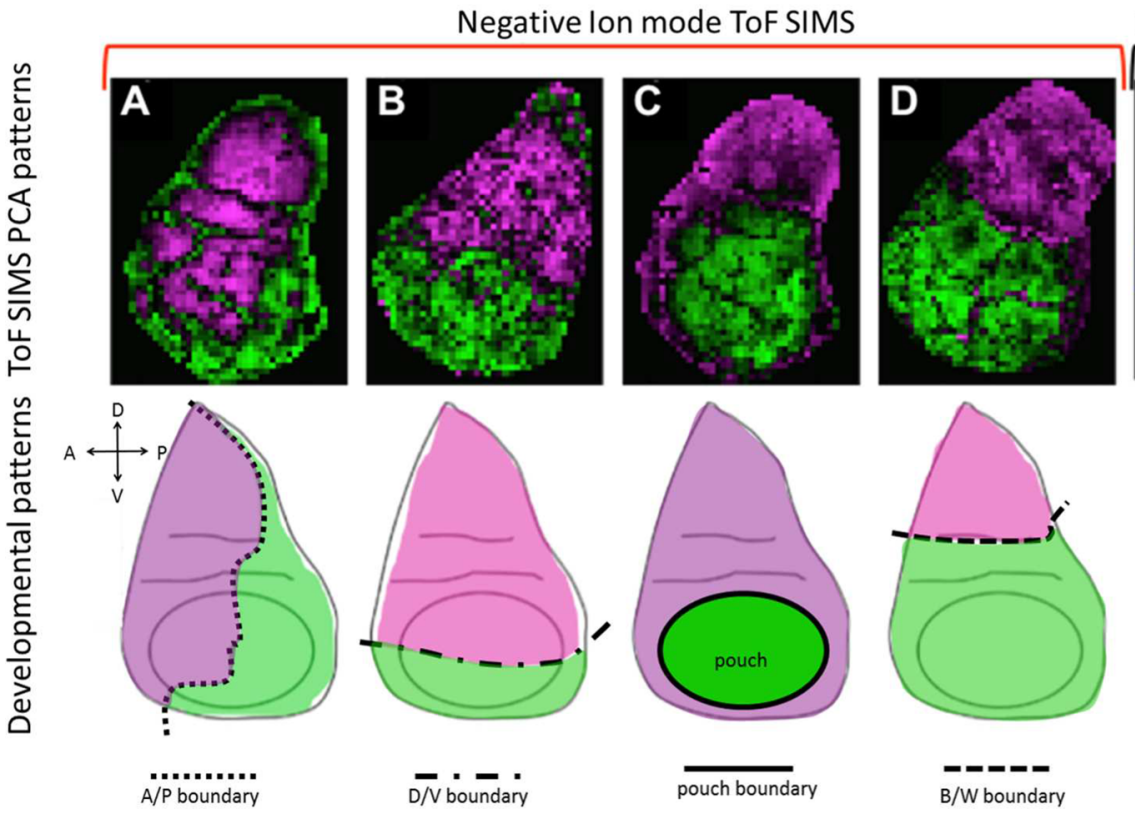

Positive lon mode

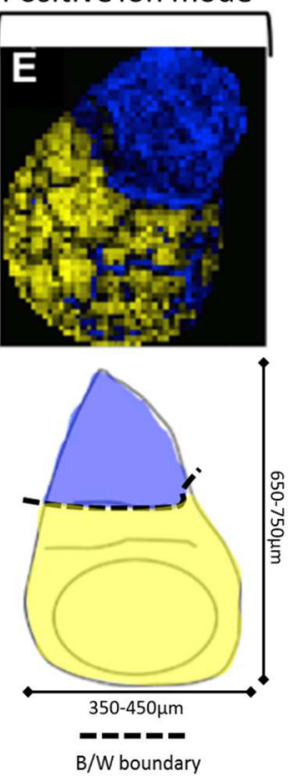

Figure 1. Small molecule distributions revealed by ToF-SIMS on the wing imaginal disc of Drosophila melanogaster (top) and corresponding developmental patterns (bottom, refs 23, 24). PCA of ToF-SIMS PCA data (top row) revealed patterns reminiscent of developmental patterns (bottom row). The patterns anterior/posterior (A/P; A), dorsal/ventral (D/V; B), pouch/nonpouch (N/NP; C), and body wall/wing blade (B/W; $\mathrm{D}$ ) were detected. The $\mathrm{A} / \mathrm{P}, \mathrm{D} / \mathrm{V}$, and $\mathrm{P} / \mathrm{NP}$ compartments were only observed in negative ion mode. The $\mathrm{B} / \mathrm{W}$ subdivision was observed in both negative and positive ion modes $(\mathrm{E}) . \mathrm{A}=$ anterior, $\mathrm{P}=$ posterior, $\mathrm{D}=$ dorsal, $\mathrm{V}=$ ventral, $\mathrm{B}=$ body wall, $\mathrm{W}=$ wing blade. Wing imaginal discs dimensions indicated.

of the developing tissue. Genes with similar restricted expression patterns are thought to play related roles. In contrast to the wealth of knowledge that has been obtained about the spatiotemporal restriction of gene expression, as well as that of the corresponding RNAs and proteoforms, the organization and distribution of other molecules, such as carbohydrates and lipids, is not well understood. This lack of knowledge can be traced back to the challenges associated with studying the localization of small molecules, e.g., lipids, with high spatial resolution. Recent work in the model system Drosophila melanogaster has described and quantified lipids over the course of its life cycle. ${ }^{12,13}$ These studies demonstrated that different tissues exhibit distinct phospholipid compositions but did not report the intraorgan distribution of these small molecules. Such spatial information is crucial to relating specific molecular moieties to specific biological processes in genetic, disease, or developmental models. In order to identify novel small molecules, such as lipids and carbohydrates, that are crucial for a developmental process, we need to look for distribution patterns that resembled those of already known components.

In this work, we determined the distribution of small molecules in the Drosophila wing imaginal disc, using a combination of imaging time-of-flight secondary ion mass spectrometry (ToF-SIMS) and coherent anti-Stokes Raman spectroscopy (CARS). These two complementary, label-free techniques make use of two inherent properties of small molecules, their mass and vibrational properties. We demonstrate that small molecules show well-defined distributions within this tissue. Strikingly, these distributions mimic genetically established patterns known to be essential for wing development and growth.

\section{MATERIALS AND METHODS}

Sample Preparation. Drosophila melanogaster were grown under standard growth conditions at $22{ }^{\circ} \mathrm{C}$. Adult flies were transferred to new food every 2 days. Wing imaginal discs were manually dissected from wild-type yellow white (yw) third instar larvae (day 6 after egg laying) for CARS and ToF-SIMS analyses unless stated otherwise. For ToF-SIMS analysis (results shown in Figures 1A and 2A,B,C), genetically modified Drosophila lines with the genotype $y w$,hsp-flp; UASmCD 8::GFP/CyO; hh-Gal4/TM6b were used. ${ }^{14}$ The $m C D 8:: G F P$ is a fusion protein between mouse lymphocyte marker CD8 and the green fluorescence protein, which is expressed under $h h$ control in this study. The hh-Gal4 is an enhancer trap line inserted into the hh locus. The enhancer trap carries a Gal4 gene and Gal4 expression is now influenced by the $h$ h regulatory elements. Wing imaginal discs were manually dissected in ice-cold phosphate-buffered saline (PBS) and dehydrated in a $10 \times$ PBS solution (Sigma-Aldrich, Zwijndrecht, $\mathrm{NL}$ ) for $30 \mathrm{~min}$. For CARS, the discs were then mounted on conventional microscopy cover slides, washed with MS-grade water (Sigma-Aldrich, Zwijndrecht, NL) three times to remove additional salts, and then analyzed.

For ToF-SIMS, discs were transferred to conductive ITO slides with $4-8$ ' $\Omega$ resistance, Delta Technologies, Stillwater, $\mathrm{MN}$ ). The discs were washed with MS grade water (SigmaAldrich, Zwijndrecht, NL) three times to remove additional salts and then air-dried. The discs were then covered with a 2 $\mathrm{nm}$ gold layer using a sputter coater (Quorums Technologies SC7640, Newhaven, UK) equipped with a FT7607 quartz crystal microbalance stage and a FT7690 film thickness monitor. For LCMS/MS analysis, a Bligh and Dyer extract was prepared from the wing discs prior to analysis. ${ }^{15}$

Microscopy. Discs were imaged with a Leica DMRX (Leica, Wetzlar, Germany) microscope equipped with an OSRAM 

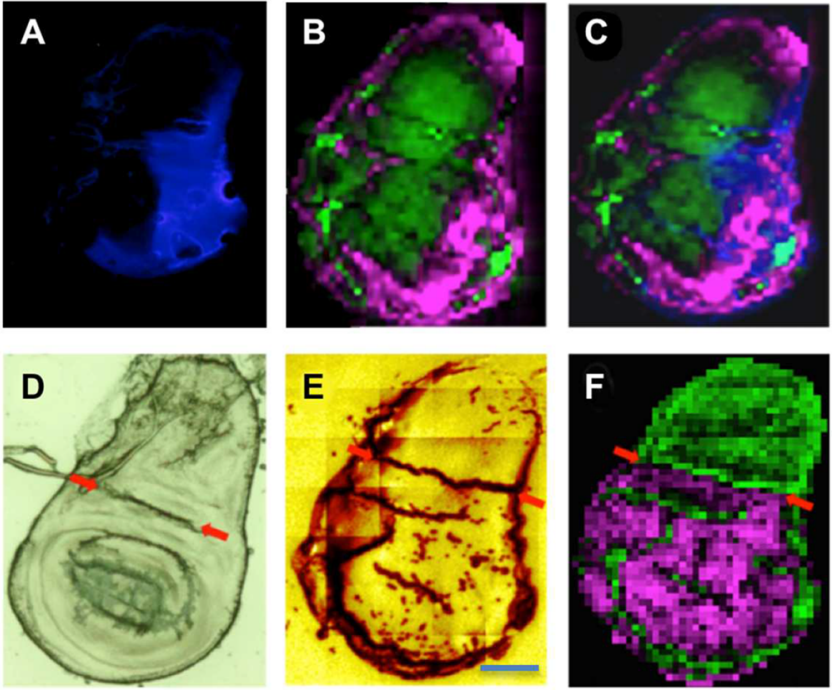

Figure 2. Small molecule patterns identified by ToF-SIMS mimic known developmental patterns. Upper panel: Imaginal disc microscopy images of GFP-labeled hedgehog expression patterns that occur exclusively in the posterior compartment (A) and ToF-SIMS PCA showing the anterior compartment (green) and posterior compartment (purple; B) of the same imaginal disc were superimposed (C). Lower panel: The fold appearing in late third instar (D, E, F, red arrows) was used as a landmark for the nonclassical body wall/wing blade $(\mathrm{B} / \mathrm{W})$ pattern. In the high vacuum of the instrument, this fold expands to a crack (E, red arrows). The expression of small molecules, determined by ToF-SIMS PCA, changes exactly at this crack boundary (F, red arrows). Thus, both boundaries revealed by PCA coincide with established compartment boundaries in Drosophila development. The scale bar in $\mathrm{E}$ indicates $150 \mu \mathrm{m}$.

HBO 50W/L2 short arc mercury lamp (Osram AG, München, Germany) at $10 \times$ magnification.

Data Acquisition. ToF-SIMS. Secondary ion mass spectrometry was performed using a Physical Electronics (PHI) TRIple focusing ToF ion (TRIFT-II) instrument equipped with a gold liquid metal ion gun (Physical Electronics, Chanhassan, MN, USA). Then, $22 \mathrm{keV} \mathrm{Au}^{+}$primary ions were "micro" focused on the sample surface. The total primary ion dose density amount was kept well below the static limit. Positive or negative secondary ions were extracted to the mass analyzer with a static voltage of $\pm 3.5 \mathrm{kV}$ and postaccelerated in front of the ion detector (dual-stage microchannel plate) by an additional $10 \mathrm{kV}$. The signal from ions in the $\mathrm{m} / \mathrm{z}$ range $1-$ $1500 \mathrm{~m} / z$ was recorded. Full wing discs were imaged step-bystep in a mosaic formed by $8 \times 8$ individual tiles between which the stage moved. ${ }^{16,17}$ Each tile of about $80-95 \mu \mathrm{m}$ in width was probed by the primary ion beam in a $256 \times 256$ pixel raster for a duration of $30 \mathrm{~s}$ to ensure that the total ion dose was below the static limit of SIMS. The size of each pixel was approximately $0.35 \mu \mathrm{m}$. The resulting image was saved as a RAW file for further data processing. The pulse width of the primary ion beam was $1 \mathrm{~ns}$.

CARS Microspectroscopy. A dual-output laser source (Leukos-CARS, Leukos, Limoges, France) provided the pump and Stokes beams to enable CARS analysis. The laser source was a passively Q-switched $1064 \mathrm{~nm}$ microchip laser, delivering $<1 \mathrm{~ns}$ pulses at a $32 \mathrm{kHz}$ repetition rate and $\sim 300 \mathrm{~mW}$ average power. The laser beam was equally divided into two separate beams with a 50/50 beam splitter. One beam was sent through a bandpass filter (FL1064-10, Thorlabs, USA) and used directly as the pump beam. The other beam was introduced into a photonic crystal fiber that creates super continuum emission of $420-2400 \mathrm{~nm}$ at the fiber output, with $>100 \mu \mathrm{W} \mathrm{nm}{ }^{-1}$ spectral power density from 1.05 to $1.6 \mu \mathrm{m}$. The supercontinuum was coupled out of the fiber with a reflective collimator (RC04APCP01, Thorlabs, USA) and passed through $700 \mathrm{~nm}$ (FEL0700, Thorlabs, USA) and $830 \mathrm{~nm}$ (LP02-830RS-25, Semrock, USA) long-pass filters. The Stokes and pump beams overlapped at a dichroic mirror (LP02-1064RU-25, Semrock, USA) and were introduced into a modified inverted microscope (Eclipse Ti-U, Nikon, Japan). The pump and Stokes pulses were tightly focused onto the sample with a near IR objective (PE IR Plan Apo 100×, NA 0.75, Seiwa, Japan). The sample was mounted on nested stepper-motor-driven (Microstage, Mad City Laboratories, USA) and piezo-driven stages (Nano-PDQ 375 HS, Mad City Laboratories, USA) that together provide $25 \mathrm{~mm}$ travel range with $<1 \mathrm{~nm}$ resolution. The CARS signal generated by the sample was collected in the forward direction by another objective (M-20X, NA 0.4, Newport, USA) and sent through notch (NF03-532/1064 $\times 10^{-25}$, Semrock, USA) and shortpass filters (FES1000, Thorlabs, USA) to remove the pump and Stokes beams. The filtered CARS beam was dispersed by a spectrometer (Shamrock 303i, 300 lines $\mathrm{mm}^{-1}, 1000 \mathrm{~nm}$ blaze, Andor, UK) and detected on a deep-depletion CCD (Newton DU920P-BR-DD, Andor, UK). The sample was raster-scanned across the focal volume with steps of $1 \mu \mathrm{m}$ in-plane and $2 \mu \mathrm{m}$ axially. Large three-dimensional images were reconstructed from adjacent tiles with in-plane dimensions of $75 \times 75 \mu \mathrm{m}$ or $50 \times 50 \mu \mathrm{m}$ and axial dimensions determined by the thickness of the wing disc due to the large dimension of the wing disc. For each position in the sample, a CARS spectrum in the range between -3400 and $-600 \mathrm{~cm}^{-1}$ was acquired. CARS images were acquired with pixel dwell times of $50 \mathrm{~ms}$.

Lipid Analysis by HPLC-ESI-MS/MS. Third instar wing imaginal discs were collected using the mass isolation approach developed in-house as described in ref 17. Approximately, 1000 individual discs were used per replicate. Lipids were extracted using a modified Bligh and Dyer method. ${ }^{15}$ The extracts were removed, dried in vacuo, and reconstituted in isopropanol. HPLC-ESI-MS/MS analyses were conducted on a Thermo Fisher Q Exactive mass spectrometer (San Jose, CA) with online separation using a Thermo Fisher/Dionex RSLC nano HPLC. HPLC conditions were as follows: Atlantis dC18, $3 \mu \mathrm{m}$, $300-\mu \mathrm{m} \times 150 \mathrm{~mm}$ column (Waters Corporation, Massachusetts); mobile phase A, acetonitrile/water (40:60) containing $10 \mathrm{mM}$ ammonium acetate; mobile phase B, acetonitrile/ isopropanol (10:90) containing $10 \mathrm{mM}$ ammonium acetate; flow rate, $6 \mu \mathrm{L} / \mathrm{min}$; gradient, $10 \% \mathrm{~B}$ to $60 \% \mathrm{~B}$ over $5 \mathrm{~min}, 60 \%$ B to $99 \%$ B over 35 min and held at $99 \%$ B for 10 min. Datadependent MS/MS scans were performed using one full MS scan $[\mathrm{m} / z$ 200-2000; 70000 resolution $(\mathrm{m} / z$ 300)] followed by fragmentation in the HCD collision cell of the six most abundant ions in the precursor scan using a normalized collision energy of 35 arbitrary units and mass analysis in the Orbitrap at 17500 resolution. Separate analyses were conducted using positive and negative ion detections.

Data analysis details for all experimental techniques have been provided in the Supporting Information.

\section{RESULTS}

Third instar wing imaginal discs were analyzed with ToF-SIMS imaging mass spectrometry in both negative and positive ion mode. Principal component analysis (PCA) was used to unravel 
the complexity of the ToF-SIMS data sets. Inspection of the principal components (PC) from the negative ion mode measurements revealed four PCs reflecting molecular distributions (Figure 1, top row), which colocalized with known genetically predicted tissue subtypes such as anterior vs posterior compartmentalization $(\mathrm{A} / \mathrm{P}$; Figure $1 \mathrm{~A})$ and dorsal from ventral tissue $(\mathrm{D} / \mathrm{V}$; Figure $1 \mathrm{~B})$. Additionally, patterns reminiscent of the pouch/nonpouch (Figure 1C) and the body wall/wing blade (B/W; Figure 1D) differentiation were observed. All these compartments are known to play an important role in Drosophila melanogaster wing development and are associated with specific gene expression patterns. ${ }^{6,9,18-22}$ Positive ion mode experiments repeatedly confirmed the $\mathrm{B} / \mathrm{W}$ distribution, and it was therefore chosen to follow up (Figure 1E and Figure S1).

Next, we determined whether two compartment boundaries (A/P and $\mathrm{B} / \mathrm{W}$ ) observed by ToF-SIMS matched with known developmental boundary markers. For the A/P subdivision, we used the classical compartment boundary established by differential expression of the morphogen hedgehog $(\mathrm{hh})$, which is responsible for the establishment and maintenance of the A/P compartment. ${ }^{25}$ Fluorescent images of third instar imaginal discs expressing GFP under the control of hh-Gal4 were generated and coregistered with the ToF-SIMS PCA score images obtained from the same imaginal discs (Figure 2A and B) (see Materials and Methods). Clearly, the subdivisions identified by ToF-SIMS were related to the compartmentalization established by the known genetic circuits (Figure 2C).

The $\mathrm{B} / \mathrm{W}$ compartment boundary (Figure $2 \mathrm{D}$, arrows) is normally delineated by a tissue fold appearing in the late third instar. ${ }^{21}$ Comparing the total ion image with the PCA image of the same imaginal disc, the fold leading to a crack in the ToF SIMS instrument provides a landmark to determine the expression pattern of small molecules observed by ToF-SIMs in the PCA (Figure 2E and F).

The analytical limitation of ToF-SIMS restricts the molecular identification of the PC patterns to small molecules. ${ }^{26} \mathrm{We}$ employed CARS to identify the main small molecule classes represented in the ToF-SIMS PCA expression patterns. CARS has the chemical specificity to allow the assignment of molecular classes. In that respect, the CARS data allow us to determine the nature of the majority of peaks and provide guidance in the selection of the patterns to investigate further. CARS provides image contrast based on molecular vibrations that are distinct for different molecules. Using CARS, we acquired the vibrational signatures of the molecules (in the frequency range corresponding to Raman shifts from -3200 $\mathrm{cm}^{-1}$ to $-900 \mathrm{~cm}^{-1}$ ) from late third instar wing imaginal discs. In the fingerprinting region $\left(-1800 \mathrm{~cm}^{-1}\right.$ to $\left.-900 \mathrm{~cm}^{-1}\right)$, the chemical moieties giving rise to specific peaks are largely known and can be found in the literature. ${ }^{27}$ Multivariate statistical analysis (PCA) was applied to screen for patterns in the CARS data set, similar to that performed for the ToF-SIMS data sets. A clear $\mathrm{B} / \mathrm{W}$ pattern similar to the one found by ToF-SIMS (Figure 3A, B, and C) is observed. No other CARS PCA pattern was observed that resembled any of the previously described patterns. Canonical correlation analysis (CCA) revealed a positive correlation score between the CARS and ToF-SIMS data sets for the $\mathrm{B} / \mathrm{W}$ patterns (Figure $3 \mathrm{C}$ and Supporting Information Table 1). Next, we investigated the spectral region of the CARS measurement from the correlating value 2 (CV2) to further identify the molecular components of the $\mathrm{B} / \mathrm{W}$ pattern. In the CARS spectra, the dispersive $\mathrm{C}-\mathrm{H}$
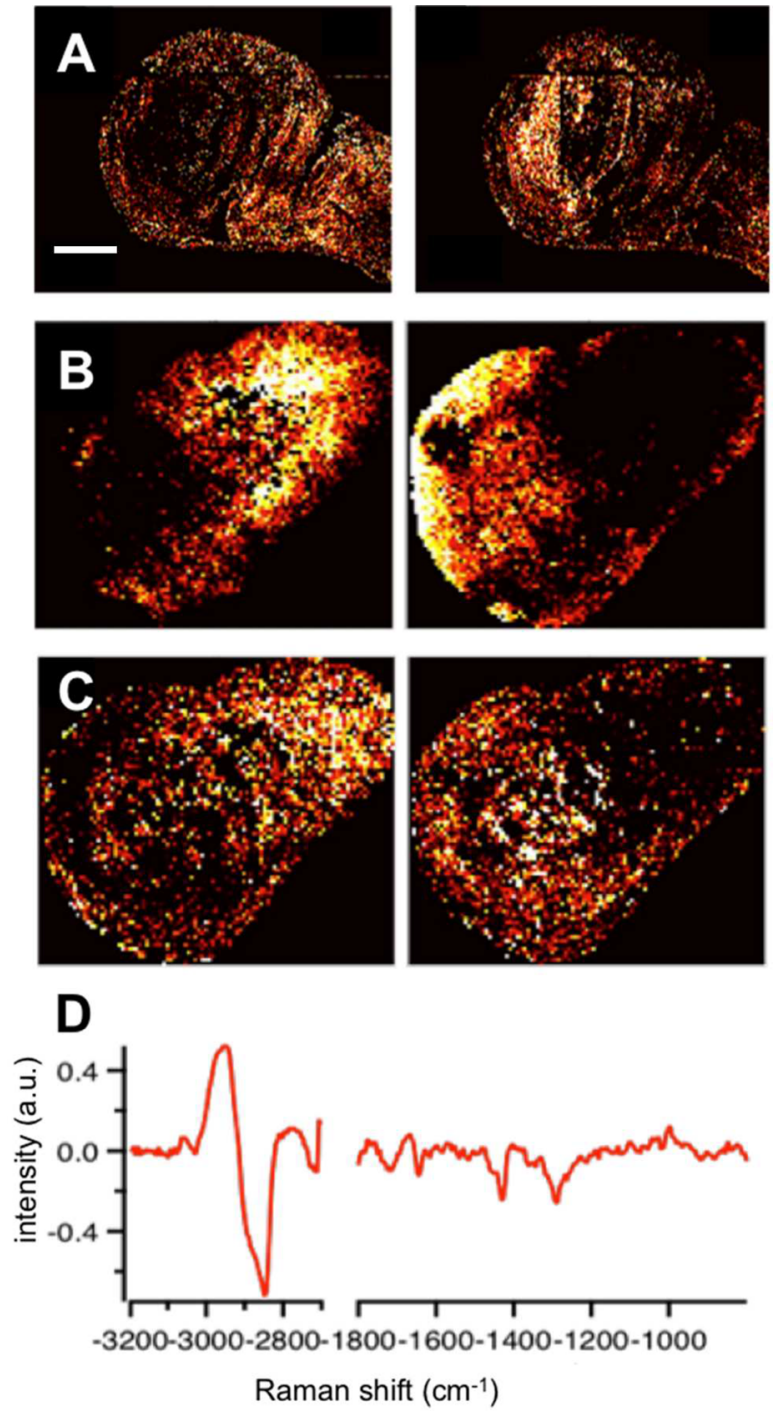

Figure 3. CARS-revealed lipid origin for the $\mathrm{B} / \mathrm{W}$ pattern. CARS was applied to test if patterns observed by ToF-SIMS can be reproduced and to gain further knowledge about the molecules involved. PCA of CARS data demonstrated a similar B/W pattern (A) to that observed in ToF-SIMS (B). CCA was applied to visualize the correlation of the observed patterns from ToF-SIMS and CARS (C; quantification listed in Supporting Information Table 1). Investigation of the normalized CARS spectra in arbitrary units (a.u.) revealed a higher content of lipids in the wing blade region compared to the body wall (D). The scale bar in A indicates $200 \mu \mathrm{m}$. The left and right images in all panels A-D represent principal components in the $3200-2700 \mathrm{~cm}^{-1}$ and the $1800-800 \mathrm{~cm}^{-1}$ wavenumber range, respectively.

feature and the phenylalanine peak at $-1000 \mathrm{~cm}^{-1}$ suggested a protein dominance for the body wall, whereas the spectral composition indicated a dominance of lipids in the wing blade section (Figure 3D). Therefore, we conclude that the components of the $\mathrm{B} / \mathrm{W}$ patterns observed in ToF-SIMS are lipids. From the ToF-SIMS spectra of the wing blade section, we observed a highly reproducible pattern of peaks. Based on previously assigned $\mathrm{m} / z$ values, 22 lipids were assigned to the classes of monoacyl- (MAGs), diacyl- (DAGs), and triacylglycerols (TAGs; Figure 4) with DAG $(34: 2 / 375.3 \mathrm{~m} / \mathrm{z})$ being the most abundant peak in the positive part of the PC loadings spectra. The principal component loading spectra show little correlation between the mono- and diacylglycerols and the 

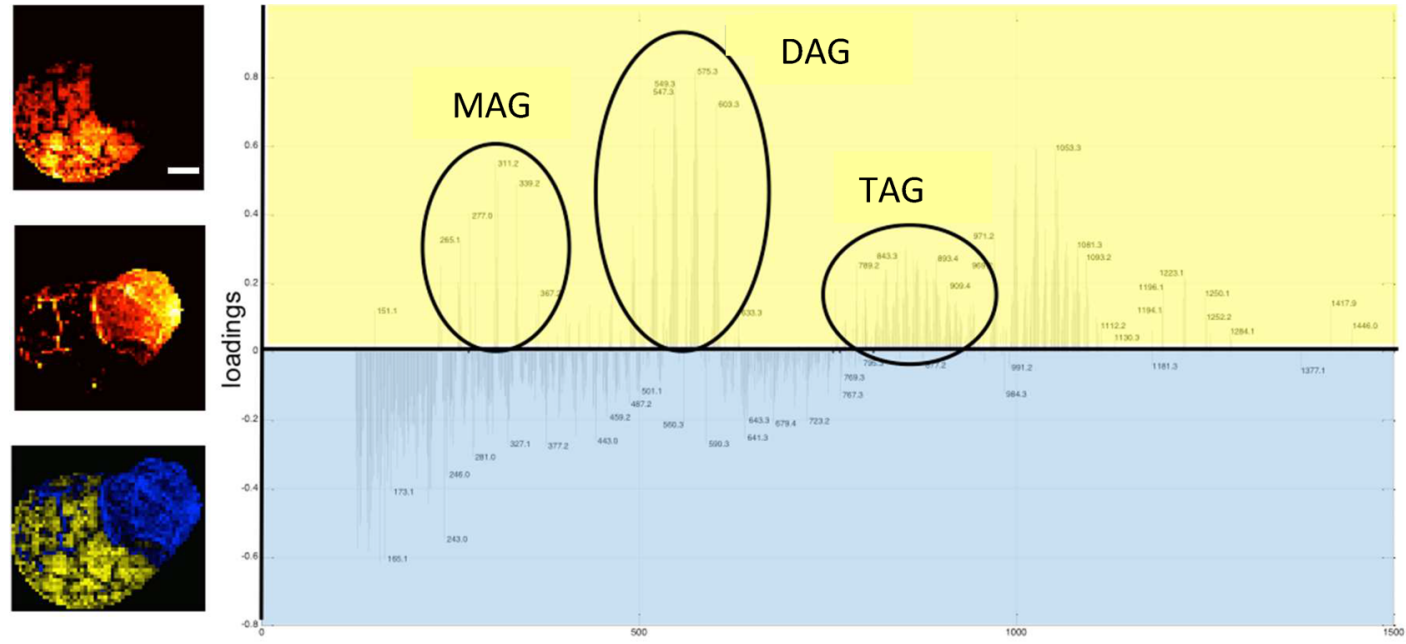

Figure 4. Assignment of peaks from ToF-SIMS measurements. The images show the positive and negative part of the PC distinguishing the B/W compartments (left top and middle panel) from the same imaginal disc as used in Figure 1. The loading spectrum on the right shows the positive loadings corresponding to $\mathrm{W}$ with a yellow background and negative loadings corresponding to $\mathrm{B}$ with a light blue background. The lower left panel is a color-coded overlay (yellow $=\mathrm{W}$, blue $=\mathrm{B}$ ). To assign the potential lipid species, we used a recently published $\mathrm{m} / z$ catalogue with assigned lipids. ${ }^{12}$ Loading spectra show that monoacyl- (MAG), diacyl- (DAG), and triacylglycerols (TAG) are over-represented in the wing blade section (yellow). The white scale bar in the top left image indicates $150 \mu \mathrm{m}$.

Table 1. Identified MAGs, DAGs, and TAGs by ToF-SIMS and MS/MS ${ }^{a}$

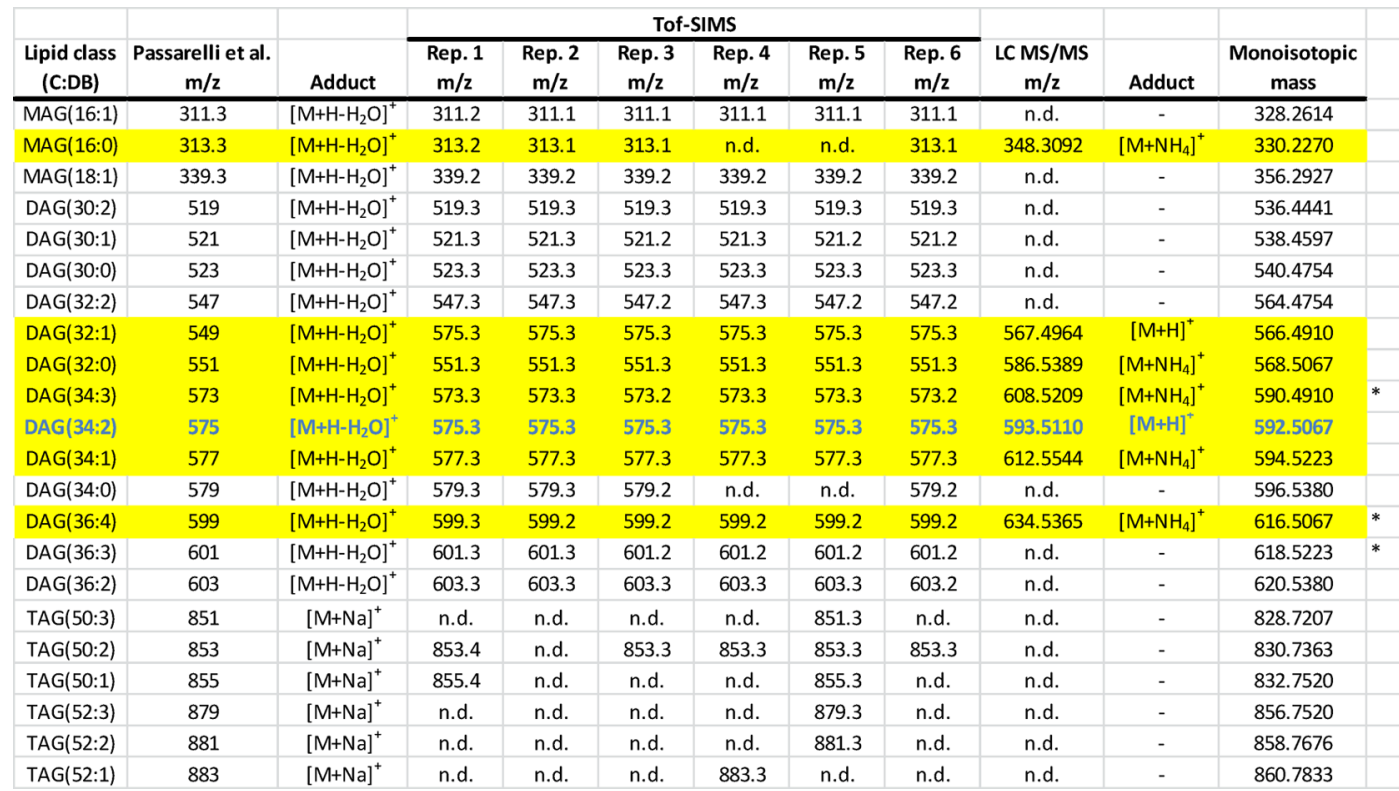

${ }^{a_{T}}$ The columns show the lipid class, mass value $(\mathrm{m} / z)$, species, as well as the monoisotopic mass of each lipid identified in the six replicate ToF-SIMS measurements (Figure S1). The lipids identified by both ToF-SIMS and MS/MS are highlighted in yellow. The most abundant peak of the ToFSIMS loading spectra $(\mathrm{m} / z$ 573.3) also identified by MS/MS is shown in blue text. n.d. indicates a non-detected species in that measurement. The asterisk in the last column indicates that the listed SIMS $\mathrm{m} / z$ values could also be explained as the sodium adduct of an ion that contains two carbon atoms and three double bonds less. For example, $\left[\mathrm{M}+\mathrm{H}-\mathrm{H}_{2} \mathrm{O}\right]$ DAG36:4 is nominally equivalent with $\left[\mathrm{M}+\mathrm{Na}-\mathrm{H}_{2} \mathrm{O}\right] \mathrm{DAG} 34: 1$.

intact phospholipids. This indicates that these peaks most likely do not originate from SIMS induced lipid fragmentation. The correlation of the MAGs and DAGs with the TAGs indicates that the acylglycerols in general play an important role in maintaining cellular organization in the wing discs. Supporting this identification is the observation of the corresponding $[\mathrm{M}+$ $\left.\mathrm{NH}_{4}\right]^{+}$DAG ions in the LC-MS/MS experiment being an indication that at least part of the overserved ions are endogenous. However, we cannot exclude that the MAG and DAG related peaks in the SIMS experiments are fragments from TAGs present on the cellular surface. The analysis conditions were optimized to minimize the fragmentation of intact phospholipids and TAGs.

To confirm this identification of the ToF-SIMS peaks, we performed orthogonal validation using high-performance liquid chromatography and electrospray ionization tandem mass spectrometry (HPLC-ESI-MS/MS, hereafter referred to as MS/MS for simplicity) of lipid extracts of whole wing imaginal disc. ${ }^{28}$ This data set was utilized as a high-mass-accuracy MS reference data set for tissue-specific lipids. We identified 156 lipid species (for details, see Materials and Methods section) containing most of the major lipid classes previously 
assigned. $^{12,13}$ Of the 156 lipid species, five were MAGs, 22 DAGs, and 10 TAGs. Seven species overlapped between the differential patterns observed by ToF-SIMS and MS/MS methods (Table 1). Among these lipids was also the previously reported DAG (34:2). Its identity in the ToF-SIMS data could further be confirmed using the loadings spectra from the negative ion mode ToF-SIMS data (Figure S2).

\section{DISCUSSION}

In this study, we applied ToF-SIMS to analyze the distribution of small molecules in the wing imaginal disc of third instar Drosophila melanogaster larvae. We found that their distributions separated into two PCs with distinct subdivisions (Figure 1). Intrigued by these ToF-SIMS patterns, we compared them to known genetic expression patterns at the same developmental time-point and found striking similarities. These pattern similarities were in part confirmed by coregistration of images of GFP fluorescence under hedgehog promotor control, a classical marker of the A/P compartment boundary. Additionally, the $\mathrm{B} / \mathrm{W}$ pattern is defined and confirmed by a well-known visible margin that correlates with the ToF-SIMS images (Figure 2). By investigating CARS PCA spectra (Figure 3 ) and tentative assignments from ToF-SIMS peaks (Figure 4), using a previously published catalogue, we identified the predominant small molecule species in the wing blade region of the $\mathrm{B} / \mathrm{W}$ pattern as lipids.

The possible role of these lipids in pattern formation and maintenance must be evaluated in light of their crucial metabolic and biochemical properties. Recent lipidomic studies revealed that DAGs and TAGs levels increase in the wing imaginal disc during larval development, reaching a peak at late third instar. ${ }^{12,13}$ While TAGs are mainly considered storage lipids from which energy can be gained via $\beta$-oxidation, ${ }^{29,30}$ they are not used for energy production during pupation and metamorphosis. ${ }^{12}$ Whether TAGs assume a different function in imaginal discs remains to be elucidated. In contrast to TAGs, DAGs show different kinetics based on their fatty acid (FA) chain length. Medium-FA-chain DAGs drastically decrease at the transition from third larval instar to the pupa stage, whereas long-chain DAGs continue to increase, which suggests that different DAGs assume distinct metabolic functions during Drosophila development; these functions also remain to be determined. We identified one DAG, termed DAG(34:2), by both ToF-SIMS and MS/MS, and found intermediate chain C16:1 and C18:1 as the two most abundant FAs in this DAG. This, and other intermediate-chain DAGs, may be used as building blocks for the rapid synthesis of new phospholipids.

Several roles of DAGs have been described. Some DAGs serve as second messengers in several receptor signal transductions via the hydrolysis of membrane lipids into DAGs and inositol-3,4,5-triphosphate $\left(\mathrm{IP}_{3}\right)$ by the enzyme phospholipase $\mathrm{C}$. The generation of $\mathrm{IP}_{3}$ leads to a release of $\mathrm{Ca}^{2+}$ from the endoplasmic reticulum. $\mathrm{Ca}^{2+}$ together with the DAG signaling pathway governs processes like cell division and differentiation. ${ }^{31}$ Additionally, phospholipase $\mathrm{D}$ activity is known to regulate cell growth and proliferation using Raf and mTOR as mediators. ${ }^{32}$ DAGs remodel the membrane in response to effector signals, which might contribute to compartment boundary formation and maintenance. In the immune system, for example, successful phagocytosis requires actin remodeling by DAG generation. ${ }^{33}$ Other mechanisms leading to similar membrane remodeling and polarization are reviewed in ref 34 . It has been demonstrated elsewhere that
DAGs in combination with other membrane lipids in distinct proportions have an important function in determining the structure and dynamics of biological membranes. ${ }^{35}$ For example, the membrane composition of a cell is important for the curvature of the membrane itself on a single cell level. ${ }^{36}$ Likewise, it can be presumed that the membrane composition of a subtissue region, such as a compartment, may influence the curvature of the tissue. Such a phenomenon might be responsible for the generation of tissue folds, and our data seem to be consistent with this notion (Figures 1 and 2). Alternatively, an over-representation of DAGs in one cell population might increase the cells' affinity to each other and thereby contribute to the segregation of cells of different populations. We hypothesize that DAGs might also be differentially distributed in the two leaflets of the lipid bilayer as a consequence of the increasing tension generated during metamorphosis. As the organism undergoes tremendous change during pupariation, it is likely that an enormous amount of membranes must be generated in a very short time. The lipid patterns observed from our studies offer important insights for the potential role(s) of lipids during Drosophila wing development and cellular organization.

\section{ASSOCIATED CONTENT}

\section{S Supporting Information}

The Supporting Information is available free of charge on the ACS Publications website at DOI: 10.1021/acs.analchem.7b00125.

Figures S1 and S2, supporting Table 1, data analysis, data spatial alignment, principal component analysis, canonical correlation analysis, and lipid analysis by HPLC-ESIMS/MS (PDF)

\section{AUTHOR INFORMATION}

\section{Corresponding Authors}

*E-mail: konrad.basler@imls.uzh.ch.

*E-mail: r.heeren@maastrichtuniveristy.nl.

ORCID

Mischa Bonn: 0000-0001-6851-8453

Ron M. A. Heeren: 0000-0002-6533-7179

\section{Present Addresses}

\#Biognosys AG, Wagistrasse 21, 8952 Schlieren, Switzerland

$\nabla$ Groupe de Spectrométries des Biomolécules et Agrégats Institut Lumière Matière Cité Lyonnaise de l'Environnement et de l'Analyze 5 rue de la Doua, 69100 Villeurbanne, France

\section{Notes}

The authors declare no competing financial interest.

\section{ACKNOWLEDGMENTS}

This project was financed in part with a grant from the Swiss SystemsX.ch initiative, grant IPP-2011/“Identification of Small Molecules Relevant for Patterning and Growth of the Wing Imaginal Disc Using High Resolution Mass Spectrometry" to E.B., K.B., and R.M.A.H. This work is part of the research program of the Stichting voor Fundamenteel Onderzoek der Materie (FOM), which is financially supported by the Nederlandse Organisatie voor Wetenschappelijk Onderzoek (NWO). R.M.A.H. acknowledges support from the Dutch province of Limburg under the LINK program. We also thank the Kanton of Zürich and the Swiss National Science Foundation for support. Special thanks to Dr. George 
Hausmann and Dr. Kerstin Euler for critical input on the manuscript and Robin Klemm for interesting discussions.

\section{REFERENCES}

(1) French, V.; Bryant, P. J.; Bryant, S. V. Science 1976, 193, 969981.

(2) Fristrom, J. W.; Mitchell, H. K. J. Cell Biol. 1965, 27, 445.

(3) Postlethwait, J. H.; Schneiderman, H. A. Dev. Biol. 1973, 32, 345-360.

(4) Gonzalezgaitan, M.; Capdevila, M. P.; Garciabellido, A. Mech. Dev. 1994, 46, 183-200.

(5) Fraser, S.; Keynes, R.; Lumsden, A. Nature 1990, 344, 431-435.

(6) Schilling, S.; Willecke, M.; Aegerter-Wilmsen, T.; Cirpka, O. A.; Basler, K.; von Mering, C. PLoS Comput. Biol. 2011, 7, e1002025.

(7) Zecca, M.; Basler, K.; Struhl, G. Cell 1996, 87, 833-844.

(8) Nellen, D.; Burke, R.; Struhl, G.; Basler, K. Cell 1996, 85, 357368.

(9) Kornberg, T. B.; Guha, A. Curr. Opin. Genet. Dev. 2007, 17, 264271.

(10) Tabata, T. Nat. Rev. Genet. 2001, 2, 620-630.

(11) Campbell, G.; Tomlinson, A. Cell 1999, 96, 553-562.

(12) Guan, X. L.; Cestra, G.; Shui, G. H.; Kuhrs, A.; Schittenhelm, R. B.; Hafen, E.; et al. Dev. Cell 2013, 24, 98-111.

(13) Carvalho, M.; Sampaio, J. L.; Palm, W.; Brankatschk, M.; Eaton, S.; Shevchenko, A. Mol. Syst. Biol. 2012, 8, 600.

(14) Lee, T.; Luo, L. Neuron 1999, 22, 451-461.

(15) Bligh, E. G.; Dyer, W. J. Can. J. Biochem. Physiol. 1959, 37, 911917.

(16) Lee, T.; Luo, L. Q. Neuron 1999, 22, 451-461.

(17) Marty, F.; Rockel-Bauer, C.; Simigdala, N.; Brunner, E.; Basler, K. Methods 2014, 68, 260-264.

(18) Zecca, M.; Struhl, G. Development 2002, 129, 1369-1376.

(19) Ng, M.; Diaz-Benjumea, F. J.; Cohen, S. M. Development 1995, 121, 589-599.

(20) Irvine, K. D.; Rauskolb, C. Annu. Rev. Cell Dev. Biol. 2001, 17, $189-214$.

(21) Diez del Corral, R.; Aroca, P.; Gomez-Skarmeta, J. L.; Cavodeassi, F.; Modolell, J. Genes Dev. 1999, 13, 1754-1761.

(22) Tabata, T.; Kornberg, T. B. Cell 1994, 76, 89-102.

(23) True, J. R. Trends Ecol Evol. 2003, 18, 640-647.

(24) Dahmann, C.; Basler, K. Trends Genet. 1999, 15, 320-326.

(25) Dahmann, C.; Basler, K. Cell 2000, 100, 411-422.

(26) Passarelli, M. K.; Winograd, N. Biochim. Biophys. Acta, Mol. Cell Biol. Lipids 2011, 1811, 976-990.

(27) Movasaghi, Z.; Rehman, S.; Rehman, I. U. Appl. Spectrosc. Rev. 2007, 42, 493-541.

(28) Gao, X. L.; Zhang, Q. B.; Meng, D.; Isaac, G.; Zhao, R.; Fillmore, T. L.; et al. Anal. Bioanal. Chem. 2012, 402, 2923-2933.

(29) Coleman, R. A.; Mashek, D. G. Chem. Rev. 2011, 111, 63596386.

(30) Athenstaedt, K.; Daum, G. Cell. Mol. Life Sci. 2006, 63, 13551369.

(31) Harden, T. K.; Sondek, J. Annu. Rev. Pharmacol. Toxicol. 2006, $46,355-379$.

(32) Hodgkin, M. N.; Pettitt, T. R.; Martin, A.; Michell, R. H.; Pemberton, A. J.; Wakelam, M. J. O. Trends Biochem. Sci. 1998, 23, 200-204.

(33) Scott, C. C.; Dobson, W.; Botelho, R. J.; Coady-Osberg, N.; Chavrier, P.; Knecht, D. A.; et al. J. Cell Biol. 2005, 169, 139-149.

(34) Almena, M.; Merida, I. Trends Biochem. Sci. 2011, 36, 593-603.

(35) Sprong, H.; van der Sluijs, P.; van Meer, G. Nat. Rev. Mol. Cell Biol. 2001, 2, 504-513.

(36) Cooke, I. R.; Deserno, M. Biophys. J. 2006, 91, 487-495. 